\title{
A topical aqueous oxygen emulsion stimulates granulation tissue formation in a porcine second-degree burn wound
}

*Jie Li, Yan-Ping Zhang, Mina Zarei, Linjian Zhu, Jose Ollague Sierra, Patricia M. Mertz, Stephen C. Davis

Department of Dermatology and Cutaneous Surgery, University of Miami Miller School of Medicine, Miami, Florida 33136, USA

* Corresponding author

Jie Li, MD, PhD, Associate Professor

Department of Dermatology and Cutaneous Surgery

University of Miami Miller School of Medicine

1600 NW $10^{\text {th }}$ Avenue, Miami, Florida 33136, USA

Phone: +1-305-243-3365, Fax: +1-305-243-6191

Email: jli@med.miami.edu

Running Title: Topical aqueous oxygen emulsion stimulates granulation tissue formation in burn wound

This study was supported by a small business innovative research grant from Defense Advanced Research Projects Agency (Davis SC) and a research grant from Dermatology Foundation of South Florida (Li J).

The authors have no conflict of interest to declare 


\begin{abstract}
Background: Oxygen is an essential substance for wound healing. Limited studies have shown that topical oxygen can influence healing. This study evaluated the effects of a topical oxygen emulsion (TOE) on burn wound healing.
\end{abstract}

Methods: A porcine second-degree burn wound model was used in the study. Burn wounds were randomly assigned to TOE, vehicle control, and no-treatment (air) groups. Effects of TOE on the granulation formation and angiogenesis were studied using Hematoxylin \& Eosin histological analysis. Protein production and gene expression of types I and III collagen and vascular endothelial growth factor (VEGF) were determined using immunofluorescent staining and Reverse Transcription and Polymerase Chain Reaction (RT-PCR), respectively.

Results: The TOE treated wounds exhibited better angiogenesis and granulation tissue formation by histology examination. The immunofluorescence staining and RT-PCR analysis demonstrated that protein production and mRNA expression of VEGF and collagen III were significantly higher in TOE treatment group than vehicle alone and air control groups, while there was no significant difference in the level of collagen I.

Conclusions: Our data demonstrates that TOE enhances burn wound healing via stimulating the expression of VEGF and type III collagen and strongly indicates the potential use of TOE in wounds.

Key words: topical aqueous oxygen emulsion; burn wound; granulation tissue formation; collagen; VEGF 


\section{INTRODUCTION}

Second-degree burn wounds involve destruction of the entire epidermis and a substantial part of the dermis and healing depends on the depth of injury. Although superficial burns can reepithelialize fairly rapid with minimal scarring, deeper second-degree and third-degree burn can take a few weeks to heal and tend to form more severe scars [1].

The tissue repair process requires an increased metabolic activity of a variety of cells, resulting in a high oxygen demand. Therefore, oxygen delivery is a critical element for the wound healing. The oxygen availability to the wound is a rate-limiting step in early repair $[2,3]$. There is a hypoxic gradient across the partially vascularized wound and the center is more hypoxic with respect to the edge and surrounding tissues. In addition, it has been shown that the percentage of revascularization correlates well with the magnitude of the hypoxic gradient [4].

A variety of studies have been shown that increased oxygen tension in a wound promotes wound healing by stimulating several processes, including collagen production [5] and blood vessel formation $[6,7]$. Different therapies have been attempted to increase local oxygen supply to wounds and accelerate wound repair such as systemic hyperbaric oxygen therapy (HBOT) and topical oxygen therapy (TOT). HBOT can be effectively applied to treat wounds, especially in hypoxic tissues [8], but it is relatively costly. Patients scheduled for HBO therapy need a careful pre-examination and monitoring. The predominant complication is represented by pressure equalization problems within the middle ear. Serious complications including barotrauma of the nasal sinuses and oxygen toxicity of the CNS rarely occur [9].

In a case-series study TOT has shown no detrimental effects on wounds and showed beneficial indications in promoting wound healing [2]. However, TOT has limited ability to 
penetrate the skin. The ideal topical oxygen agent would provide sufficient quantities of oxygen to a wound after application and be non-toxic to the skin to accelerate local tissue repair [10]. Topical Oxygen Emulsion (TOE) is a relative new technique which delivers emulsion-containing supersaturated oxygen to a wound and slowly releases additional oxygen. This technology is based on perfluorocarbon droplets being encapsulated within an aqueous continuous phase and has a high oxygen carrying capacity [11].

Our earlier porcine in vivo study found that TOE significantly enhanced the rate of wound re-epithelialization in porcine second-degree burns using a salt-split technique [11]. In this assay, the wounds with surrounding normal skin were excised and incubated in $0.5 \mathrm{M}$ sodium bromide at $37^{\circ} \mathrm{C}$ for 24 hours, allowing for a separation of the dermis from the epidermis. After the separation, the epidermal sheet was examined macroscopically for defects. The defect was defined as a hole in the area of the wound. Reepithelialization is considered complete if no defect is present or vice versa. However, the effects and mechanisms of TOE on burn wound healing process are still unknown. In this study, we evaluated the effects of TOE on the dermal angiogenesis and granulation tissue formation in porcine second-degree burn wounds. The potential mechanisms involved were explored by measuring the gene expression level with Reverse Transcription and Polymerase Chain Reaction (RT-PCR) analysis of types I and III collagens and vascular endothelial growth factor (VEGF) and their protein production using immunofluorence staining techniques. 


\section{MATERIALS AND METHODS}

Topical aqueous oxygen emulsion (TOE) and vehicle only emulsion were provided by TherOx Inc. (Irvine, California, USA) prior to the use. TOE contains super-saturated oxygen which can be delivered topically to a wound $[11,12]$. The TOE formulation is based on perfluorocarbon droplets being encapsulated within an aqueous continuous phase allowing slow release of oxygen over time. The vehicle is a proprietary oil in water perfluorocarbon emulsion [12]. The oxygen solubility of the perfluorocarbon is relatively high. The dissolved oxygen concentration contained in the topical emulsion is approximately $2.0 \mathrm{ml}$ of $\mathrm{O} 2$ (standard temperature and pressure) per $\mathrm{ml}$ of emulsion prior to dispersion, which is twenty times greater than water. The oxygen is dissolved into the perfluorocarbon emulsion and stored under pressure in a small dispensing bottle. By maintaining pressure on the emulsion, dissolution and outgassing are prevented during storage and the maximum oxygen concentration is delivered on dispensation. Upon topical applying, the oxygen released from the emulsion sustained for up to 12 hours above the atmosphere measured by a transcutaneous oxygen monitor. The topical cream is formulated with biocompatible emulsifying agents to ensure adequate stability of the dispersed perfluorocarbon droplets $[11,12]$.

\section{Animals, burn wounds, treatments and sample collections}

Pigs are an excellent animal model for the evaluation of therapeutic agents for the skin repair due to the similarity in skin histology with humans. There is a strong correlation between pig and human wound-healing studies [13]. Six white, specific pathogen free pigs were used as welldefined porcine models for this study [13]. The study was conducted in compliance with the 
University of Miami's Standard Operating Procedures and approved by the University of Miami Institutional Animal Care and Use Committee [11].

Deep partial thickness second-degree burn wounds, $8.5 \mathrm{~mm}$ in diameter by $0.8 \mathrm{~mm}$ deep, were made on the anterior two-thirds of each animal skin using cylindrical brass rods which were heated in a boiling water bath to $100^{\circ} \mathrm{C}$. Steam burn on the skin was prevented by drying the rod. The brass rod was held at a vertical position on the skin for six seconds, with all pressure supplied by gravity [11]. Immediately after burning, the roof of the burn blister was removed with a sterile spatula. The burn wounds were made approximately $2 \mathrm{~cm}$ from each other and were then randomly assigned to one of three treatment groups: 1) topical aqueous oxygen emulsion (TOE) which contains super-saturated oxygen, 2) topical vehicle only control (Ctr), and 3) no-treatment (air). TOE and vehicle only emulsion were provided in blinded pressurized containers (TherOx Inc., Irvine, California, USA). The wounds were treated with $200 \mathrm{mg}$ of TOE or vehicle only emulsion or no-treatment twice daily for the first 5 days and once thereafter for a total of 21 days.

On day 0 (prior to wounding) and days 1, 4, 7, 10,14 and 21 after wounding, excisional biopsies were taken from each treatment group. A total of 114 samples, including six unwounded skin biopsies at day 0 and six wounds (one from each pig) from each treatment at each time point, were collected. Each biopsy was taken in the center of the wounds with adjacent normal skin on each side and divided into three parts. One third of each specimens were immediately placed in cold phosphate buffered saline (PBS) then embedded into Optimal Cutting Temperature (OCT) embedding media and stored in $-80 \mathrm{C}^{\circ}$ for immunofluorescence staining analysis; one third of each wounds (only wound portion) were snap frozen in liquid nitrogen and stored in $-80 \mathrm{C}^{\circ}$ for RNA analysis; and the remaining thirds were fixed in $10 \%$ 
neutral buffered formalin for histological evaluation.

\section{Transcutaneous oxygen tension measurements in vivo}

On day 4 and day 7 post-wounding prior to the new treatment, two sites from each treatment group from two pigs were wiped with sterile saline and measurements of subcutaneous oxygen pressure (PO2) were made with a Radiometer TCM transcutaneous oxygen monitor ( Danaher Co., Washington, DC). This device consists of a Clark type polorgraphic surface probe covered by a disposable oxygen-permeable membrane. The probe was used by attaching a self-adhesive, circular fixation ring to the skin surface. Several drops of a contact solution, as supplied by the maker of the device, were place in the fixation ring to provide contact between the skin surface and the membrane of the probe. The device was calibrated to atmospheric pressure and ambient humidity and operated in accordance with the operation manual.

\section{Histological analysis}

Formalin-fixed tissues were embedded in the paraffin, cut into $5 \mu \mathrm{m}$ sections and stained with hematoxylin and eosin (H\&E). Specimens were evaluated under a Zeiss Axiovert 200

microscope with a digital camera (Carl Zeiss MicroImaging, Thornwood, NY) by a dermatopathologist blinded to the treatment groups. A semi-quantitative scoring system was used to evaluate the following elements of wound healing: 1) angiogenesis: new vessel ingrowth was scored as: $1=$ absent, $2=$ mild, $3=$ moderate, $4=$ marked, $5=$ =xuberant; 2 ) granulation tissue formation: the percentage of the granulation tissue filled the dermal defects, which consists of newly formed blood vessels, proliferating fibroblasts and newly deposited matrix collagens in wound bed. Mean scores: $0=<5 \%, 1=6-25 \%, 2=26-50 \%, 3=51-70 \%, 4=71-$ 
$90 \%, 5=91-100 \%$.

\section{Immunofluorescent (IF) staining analysis of protein productions}

The frozen tissues were cut into $6 \mu \mathrm{m}$-thick sections and fixed with cold methanol. Sections were then stained with primary antibodies of mouse monoclonal anti-type I collagen (Sigma-Aldrich, St. Louis, MO), rabbit polyclonal anti-type III collagen (Abcam, Cambridge, MA) or rabbit polyclonal anti-VEGF (Santa Cruz Biotech, Santa Cruz, CA) respectively, followed by FITCconjugated secondary antibody staining (Sigma-Aldrich). A Zeiss Axiovert 200 fluorescent microscope (Carl Zeiss, Thornwood, NY) with a digital camera was used to capture the images. The intensity of staining was quantified with image analysis software Axiovision (Carl Zeiss) and expressed as relative green density (the density was measured as green value per pixel or square picture element). A total of 18 areas were analyzed for each tissue sample (two sections were made for each sample, three pictures were taken for each section, and three areas were analyzed for each picture).

\section{RNA isolation and RT-PCR reaction}

Total RNAs were isolated from samples using RNA isolation kit (Qiagen, Germantown, MD). The concentration of RNA was measured using spectrophotometer (Beckman, Indianapolis, IN). Then using the specific primers, the mRNA abundance was measured for porcine collagen I, collagen III and VEGF and $\beta$-actin (primers for each gene see Table 1) by Reverse Transcription and Polymerase Chain Reactions (RT-PCR) with RT-PCR kit (Qiagen). To amplify $\beta$-actin, collagen I and collagen III, cDNA was synthesized from RNA by reverse transcription for 30 $\min$ at $50^{\circ} \mathrm{C}$, then cDNA was amplified with following procedure: Denaturation, at $95^{\circ} \mathrm{C}$ for 15 
min, then repeat 34 cycles of denaturation at $94^{\circ} \mathrm{C}$ for $30 \mathrm{sec}$, annealing at $65^{\circ} \mathrm{C}$ for 1 min and extension at $72^{\circ} \mathrm{C}$ for $90 \mathrm{sec}$, followed with final extension at $72^{\circ} \mathrm{C}$ for $8 \mathrm{~min}$. To amplify VEGF, cDNA was synthesized from RNA by reverse transcription for $45 \mathrm{~min}$ at $48^{\circ} \mathrm{C}$, then cDNA was amplified with following procedure: Denaturation, at $95^{\circ} \mathrm{C}$ for $15 \mathrm{~min}$, then repeat 28 cycles of denaturation at $94^{\circ} \mathrm{C}$ for $30 \mathrm{sec}$, annealing at $60^{\circ} \mathrm{C}$ for $1 \mathrm{~min}$ and extension at $68^{\circ} \mathrm{C}$ for $2 \mathrm{~min}$, followed with final extension at $68^{\circ} \mathrm{C}$ for $7 \mathrm{~min}$. PCR reactions were performed for each sample in duplicate and the PCR products were run on $2 \%$ agarose gel in duplicate. As an internal control, $\beta$-actin (a house keeping gene) RT-PCR products were added to each of RT-PCR products of collagen I, collagen III and VEGF before loading to gel. After running, the gel was stained with ethedium-bromide and visualized under UV light with BioRad Gel Document 2000 instrument. The intensity of the bands was analyzed with Bio-Rad Quantity-One software. The relative expression level for collagen I, collagen III and VEGF was divided by the density of its respective $\beta$-actin band for normalization. The resulted value was expressed as the percentage of normal value.

\section{Statistic analysis}

GraphPad Prism version 5.0 (GraphPad Software Inc., San Diego, CA) was used for statistical analysis. Data were analyzed using two-way analysis of variance (ANOVA) followed by an unpaired two-tailed Student's $t$-test. P value $<0.05$ was considered significant. 


\section{RESULTS}

\section{TOE treatment increased oxygen tension in pig skin dermis}

Measured by a transcutanous oxygen tension $\mathrm{PO} 2$ monitor the oxygen released from the emulsion sustained for more than 12 hours above the atmosphere. Two-time higher oxygen levels in pig dermis were recorded in the TOE treatment group compared with vehicle alone and no-treatment groups, with mean $\mathrm{PO} 2$ tension measurements about $25 \mathrm{mmHg}$ and $22 \mathrm{mmHg}$ at day 4 and day 7 for TOE group compared with $12 \mathrm{mmHg}$ and $10 \mathrm{mmHg}$ for vehicle only or notreated controls.

\section{TOE stimulated burn wound angiogenesis and granulation tissue formation}

Dermal repair is characterized by granulation tissue formation consisting mainly of two phenomena of angiogenesis and fibroplasia that repair the dermal defect. The later is characterized with proliferating fibroblasts and deposition of extracellular matrix. New blood vessel formation or angiogensis plays important role in providing nutrients to the wounded area and healing of the wound. The burns that were treated with the active TOE exhibited better angiogenesis in all time points examined as compared to vehicle alone and untreated burn wounds, significant higher on day 10 compared with vehicle control (p $<0.05$ ) and untreated wounds ( $\mathrm{P}<0.001)$, respectively (see Fig. 1A). The burns that were treated with the active TOE exhibited better granulation tissue formation during the entire experiment, with significant higher scores at day 10 as compared to vehicle alone $(\mathrm{P}<0.05)$ and untreated burns $(\mathrm{P}<0.01)$ (Fig. 1B). There is good correlation between the granulation tissue formation and the angiogenesis findings (Fig. $1 \mathrm{~A}$ and $1 \mathrm{~B})$.

\section{TOE stimulated VEGF protein production and mRNA expression}

To understand the potential mechanism involved in the enhanced wound angiogenesis, we 
sought to analyze the VEGF expression since it is a major growth factor for endothelial cells which are the critical for wound angiogenesis. The immunofluorescence (IF) analysis demonstrated that the protein production of VEGF was increased in the early phase of wound healing in all the treatments. VEGF protein expression showed higher level at days 1, 4, 7 and 10 in all the treatments (Fig. 2A and 2B), however, the level of VEGF protein production was significantly higher in TOE treatment group. As shown in Fig. 2A and 2B, the TOE treatment marked increased the protein level of VEGF at day 1 compared with vehicle alone $(\mathrm{p}<0.05)$ and untreated control $(\mathrm{p}<0.01)$ and at day 4 compared with vehicle $(\mathrm{p}<0.05)$ and untreated controls ( $\mathrm{p}<0.001)$, respectively.

Similar as the protein production, the RT-PCR analysis showed that the expression of VEGF mRNA was increased starting as early as day 1 , reached its peak at day 4 , and returned to normal level at day 14 post wounding. Compared with vehicle alone and untreated controls, the significant higher level expressions in TOE treated wounds were observed at day 4 ( $\mathrm{P}<0.01, \mathrm{P}$ $<0.05$ ) and day 7 (both $\mathrm{P}<0.01$ ) (Fig. $2 \mathrm{C}$ and 2D).

\section{TOE increased collagen protein production and mRNA expression}

Type I and type III collagens are the major collagens in skin dermis and their productions are dramatically increased during the early phase of wound repair. The effects of TOE on protein productions and gene expressions of type I and III collagens were determined with IF staining and semi-quantitative RT-PCR respectively.IF staining revealed the increased protein production of collagen III started at day 7 and reached peak at day 14 post wounding. As shown in Fig. 3A and 3B, a significant higher level of collagen III protein was observed in wounds treated with TOE at day 10 and day 21 compared with vehicle control $(\mathrm{p}<0.01, \mathrm{p}<0.05)$ or untreated control 
(both $\mathrm{p}<0.05$ ). RT-PCR results also showed significantly increased mRNA expression of collagen III in burn wounds treated with TOE compared with wounds treated with vehicle alone or no treatment (air). Higher expression level of collagen III mRNA occurred with a peak at day 10 through day 21 in wounds treated with TOE, with significant higher value at day 21compared with vehicle and untreated controls (both $\mathrm{p}<0.05$ ) (Fig. 3C and 3D).

For type I collagen, its expression was markedly increased from day 10 through day 21 of post wounding in all the burn wounds with different treatments. However, no significant difference was detected between TOE treated wounds and two control groups (data not shown). 


\section{DISCUSSION}

The tissue repair process requires an increased metabolic activity of a variety of cells, resulting in a high oxygen demand [15]. Injured skin causes impaired blood vessel and result in hypoxia and ischemia in the wounds. Although the initial hypoxia in the wounded area can stimulate collagen production [16] and angiogenesis [17], long-term lacking oxygen in the wounds impedes wound healing. Providing oxygen would improve wound healing. HBOT and TOT have been shown promising effects in revascularization and re-epithelialization of wounds. But their applications are limited by high cost or low ability to penetrate the skin [11]. TOE can overcome these limitations $[11,18]$ and our results showed that TOE stimulated the healing of secondary burn wounds.

The restoration of dermis is essential for the reestablishment of skin integrity and function. Granulation tissue formation is the hallmark of dermal repair and characterized by angiogenesis and collagen deposition in morphology. Angiogenesis includes endothelial cell proliferation, migration and neovascularization by sprouting from pre-existing vessels and is essential for the timely healing. Newly formed blood vessels participate in granulation tissue formation and provide nutrition and oxygen for growing tissues. Hopf et al. showed a stimulation of neovascularization in hypoxic tissue and also increased vascular endothelial growth factor (VEGF) levels after HBO treatment [7, 19]. Similarly, our histology analysis found that TOE significantly increased angiogenesis of second-degree burn wounds compared with burn wounds treated with vehicle and air control.

VEGF is a key growth factor which mediates angiogenesis [20, 21]. VEGF is expressed at low level in intact skin, but its expression is up-regulated during wound healing. Low oxygen tension during tissue injury is one of major inducers of VEGF [22]. In this study TOE treatment 
significantly increased the VEGF mRNA expression and protein production from day 1 to day 10 of post-wounding (Fig. 2). The positive effects of TOE on wound healing through regulating the expression of VEGF was also observed in the chronic wounds. Gordillo et al. observed higher VEGF expression in the wound edge tissues when the human chronic wounds were treated with topical oxygen therapy [23]. Several other studies also found that oxygen administration increased VEGF protein expression in wound fluids in vivo [19, 24, 25].

Type I and type III collagens are the major interstitial fiber-forming collagens in normal human dermis. Oxygen plays a critical role in collagen synthesis and tensile strength of wounds. Wound environments that are anoxic inhibit collagen cross-linking because oxygen is a necessary co-factor. A clinical investigation found that the amount of deposited collagen was proportional to the $\mathrm{pO} 2$ value present in the wound. In that study, Jonsson et al. measured the oxygen tension and collagen deposition in standardized, subcutaneous wounds in 33 postoperative surgical patients. These results demonstrated that collagen deposition was directly and significantly proportional to wound oxygen tension and measure of perfusion [26]. In another study, Siddiqui et al. found that the collagen synthesis rate of human fibroblasts was decreased under chronic hypoxia in vitro [27]. Also, the study by Gordillo et al. found that portable topical oxygen therapy improved healing of human chronic wounds [23]. Consistent with the above studies, we found that TOE significantly increased mRNA expression and protein level of type III collagen in the burn wounds compared with ones treated with vehicle control or no-treatment (air).

Type III collagen is the predominant collagen synthesized during the early stage of wound healing. During normal excisional wound repair, type III collagen first appears 48 to 72 hours after wounding and is maximally secreted between 5 and 7 days of post wounding. During 
wound repair, a gradual turnover of collagen occurs: type I collagen synthesis increases while type III collagen undergoes degradation. In this study our immunofluorescent results showed that type III collagen protein reached highest level at day 10 and started to decrease at day 14 . TOE significantly increased the protein level of collagen III at day 10 and day 21 compared to burn wounds treated with vehicle and air control (Fig. 3A and 3B). Our RT-PCR analysis also demonstrated marked increase of type III collagen expression in the wounds treated with TOE from day 10 through day 21 (Fig. 3C and 3D). Our data suggest that the enhancing effects of TOE on wound healing are related to increased protein production of type III collagen in the wounds. Collagens are important for granulation tissue formation in the wounds. Correspondent with the stimulatory effects of TOE on collagen III production, our histology analysis also found that TOE increased wound granulation tissue formation compared to vehicle and air control (Fig. 1).

Type I collagen becomes predominant in the later stage of granulation tissue formation and remained as most abundant collagen component (more than 80\%) in the scar tissue in the newly formed dermis. Earlier studies have shown a close correlation of scar formation with increased expression of type I collagen and the reverse correlation with the elevated expression of type III collagen $[28,29]$. Interestingly, our study could not detect the significant increase of type I collagen expression in burn wounds treated with TOE. Our finding may indicate potential significance of TOE treatment in the scar formation in burn wounds via stimulating the expression of type III collagen without affecting type I collagen. However, further studies with more samples and with third-degree burn wounds are needed to verify this hypothesis.

Our results of protein and mRNA analysis have demonstrated that the promoting effects of TOE on wound healing may be related to its effects on improving angiogenesis and 
granulation tissue formation in the wounds. Our histology analysis also found TOE stimulated granulation tissue formation and angiogenesis. There is a good correlation between the angiogenesis and the granulation tissue formation in histology (Fig. 1A and 1B), as well as a good correlation between histological findings and the protein and mRNA expression levels of VEGF (Fig. 2) and type III collagen (Fig. 3).

Our results are in agreement with previous reports. Wu et al. found significantly impaired epithelial ingrowth and granulation tissue deposition under ischemia in an ischemic ulcer model [30]. HBO treatment showed to increase the production of new granulation tissue in an ischemic rabbit ear ulcer model [31]. Our recent study with a porcine deep-partial thickness wound model showed that TOE stimulated production of VEGF and both type I and type III collagen as well [32]. 


\section{CONCLUSION}

In summary this study shows that topical active oxygen emulsion applications are able to enhance the healing of second-degree burns as compared to vehicle and air controls. The positive effects can be attributed to the sustained high level of oxygen released in wounds by TOE and its stimulating effects of type III collagen and VEGF production. Our results indicate the potential application of this topical oxygen emulsion in burns and ischemia affected slow or non-healing wounds in clinical setting. 


\section{ACKNOWLEDGEMENTS}

This study was supported by a small business innovative research grant from Defense Advanced

Research Projects Agency (Davis SC) and a research grant from Dermatology Foundation of South Florida (Li J). We thank Dr. Ling Tang for her help with the manuscript. 


\section{LIST OF ABBREVIATIONS}

CNS: Central Nervous System

HBOT: Hyperbaric Oxygen Therapy

H\&E: hematoxylin and eosin

IF: Immunofluorescent

PBS: Phosphate Buffered Saline

PO2: oxygen pressure

OCT: Optimal Cutting Temperature

RT-PCR: Reverse Transcription and Polymerase Chain Reaction

TOE: Topical Oxygen Emulsion

TOT: Topical Oxygen Therapy

VEGF: Vascular Endothelial Growth Factor 


\section{REFERENCES}

[1] Monstrey S, Hoeksema H, Verbelen J, Pirayesh A, Blondeel P. Assessment of burn depth and burn wound healing potential. Burns 2008;34:761-9.

[2] Kalliainen LK, Gordillo GM, Schlanger R Sen CK. Topical oxygen as an adjunct to wound healing: a clinical case series. Pathophysiology 2003:9:81-87.

[3] Sen CK. Wound healing essentials: let there be oxygen. Wound Repair Regen 2009;17:1-18.

[4] Remensnyder JP, Majno G. Oxygen gradients in healing wounds. Am J Pathol 1968; 52:301-23.

[5] Hsu RW, Hsu WH, Tai CL, Lee KF. Effect of hyperbaric oxygen therapy on patellar tendinopathy in a rabbit model. J Trauma 2004;57:1060-4.

[6] Mader JT, Brown GL, Guckian JC, Wells CH, Reinarz JA. A mechanism for the amelioration by hyperbaric oxygen of experimental staphylococcal osteomyelitis in rabbits. $\mathbf{J}$ Infect Dis 1980;142:915-22.

[7] Hopf HW, Gibson JJ, Angeles AP, Constant JS, Feng JJ, Rollins MD, et al. Hyperoxia and angiogenesis. Wound Repair Regen 2005;13:558-64.

[8] Hollander DA, Hakimi MY, Hartmann A, Wilhelm K, Windolf J. The influence of hyperbaric oxygenation (HBO) on proliferation and differentiation of human keratinocyte cultures in vitro. Cell Tissue Bank 2000;1:261-9.

[9] Plafki C, Peters P, Almeling M, Welslau W, Busch R. Complications and side effects of hyperbaric oxygen therapy. Aviat Space Environ Med 2000;71:119-24.

[10] Dimitrijevich SD, Paranjape S, Wilson JR, Gracy RW, Mills JG. Effect of hyperbaric oxygen on human skin cells in culture and in human dermal and skin equivalents. Wound 
Repair Regen 1999;7:53-64.

[11] Davis S, Cazzaniga A, Ricotti C, Zalesky P, Hsu LC, Creech J et al. Topical Oxygen Emulsion: A Novel WoundTherapy. Arch Dermatol 2007:143:1252-6.

[12] Spears JR. Stabilized gas-enriched and gas supersaturated liquids. United States Patent 6,344,489, 2002.

[13] Sullivan TP, Eaglstein WH, Davis SC, Mertz PM. The pig as a model for human wound healing. Wound Repair Regen 2001;9:66-76.

[14] Davis SC, Eaglstein WH, Cazzaniga AL, Mertz PM. An octyl-2-canoacrylate formulation speeds healing of partial thickness wounds. Dermatol Surg. 2001;27:783-8. [15] Zamboni WA, Browder LK, Martinez J. Hyperbaric oxygen and wound healing. Clin Plast Surg 2003;30:67-75.

[16] Falanga V, Zhou L, Yufit T. Low oxygen tension stimulates collagen synthesis and COL1A1 transcription through the action of TGF-1. J Cell Physiol 2002;191:42-50. [17] Li J, Zhang YP, Kirsner R. Angiogenesis in wound repair: angiogenic growth factors and the extracellular matrix. Microsc Res Tech 2003;60:107-14.

[18] Roe DF, Gibbins BL, Ladizinsky D. Topical Dissolved Oxygen Penetrates Skin: Model and Method. J Surg Res 2010:159:e29-36.

[19] Sheikh AY, Gibson JJ, Rollins MD, Hopf HW, Hussain Z, Hunt TK. Effect of hyperoxia on vascular endothelial growth factor levels in a wound model. Arch Surg 2000;135:1293-7. [20] Senger DR, Claffey KP, Benes JE, Perruzzi CA, Sergiou AP, Detmar M. Angiogenesis promoted by vascular endothelial growth factor: regulation through alpha1beta1 and alpha2beta1 integrins. Proc Natl Acad Sci USA 1997;94:13612-7.

[21] Gerber HP, Dixit V, Ferrara N. Vascular endothelial growth factor induces expression 
of the antiapoptotic proteins Bcl-2 and A1 in vascular endothelial cells. J Biol Chem 1998; 273:13313-6.

[22] Detmar M, Brown LF, Berse B, Jackman RW, Elicker BM, Dvorak HF et al. Hypoxia regulates the expression of vascular permeability factor/vascular endothelial growth factor (VPF/VEGF) and its receptors in human skin. J Invest Dermatol 1997;108:263-8.

[23] Gordillo GM, Roy S, Khanna S, Schlanger R, Khandelwal S, Phillips G et al. Clin Exp Pharmacol Physiol 2008;35:957-64.

[24] Darrington RS, Godden DJ, Park MS, Ralston SH, Wallace HM. The effect of hyperoxia on the expression of cytokine mRNA in endothelial cells. Biochem Soc Trans 1997;25:S292. [25] Maniscalco WM, Watkins RH, Finkelstein JN, Campbell MH. Vascular endothelial growth factor mRNA increases in alveolar epithelial cells during recovery from oxygen injury. Am J Respir Cell Mol Biol 1995;13:377-86.

[26] Jonsson K, Jensen JA, Goodson WH III, Scheuenstuhl H, West J, Hopf HW et al. Tissue oxygenation, anemia, and perfusion in relation to wound healing in surgical patients. Ann Surg 1991;214:605-13.

[27] Siddiqui A, Galiano RD, Connors D, Gruskin E, Wu L, Mustoe TA. Differential effects of oxygen on human dermal fibroblasts: acute versus chronic hypoxia. Wound Repair Regen $1996 ; 4: 211-8$.

[28] Volk SW, Wang Y, Mauldin EA, Liechty KW, Adams SL. Diminished type III collagen promotes myofibroblast differentiation and increases scar deposition in cutaneous wound healing. Cells Tissues Organs 2011;194:25-37.

[29] Qiu L., Jin X., Xiang D., Fu Y., Tian X. A study on collagen constitute and affected factors in hypertrophic scar at different age periods. Ann Burns Fire Disasters 2003;16:1-6. 
[30] Wu L, Xia YP, Roth SI, Gruskin E, Mustoe TA. Transforming growth factor-beta1 fails to stimulate wound healing and impairs its signal transduction in an aged ischemic ulcer model: importance of oxygen and age. Am J Pathol 1999;154:301-9.

[31] Zhao LL, Davidson JD, Wee SC, Roth SI, Mustoe TA. Effect of hyperbaric oxygen and growth factors on rabbit ear ischemic ulcers. Arch Surg 1994;129:1043-9.

[32] Li J, Ollague Sierra J, Zhu L, Tang L, Rahill K, El-Sabawi B et al. Effects of a topical aqueous oxygen emulsion on collagen deposition and angiogenesis in a porcine deep partialthickness wound model. Exp Dermatol 2013;22(10):674-6 


\section{Figure 1}

(A)

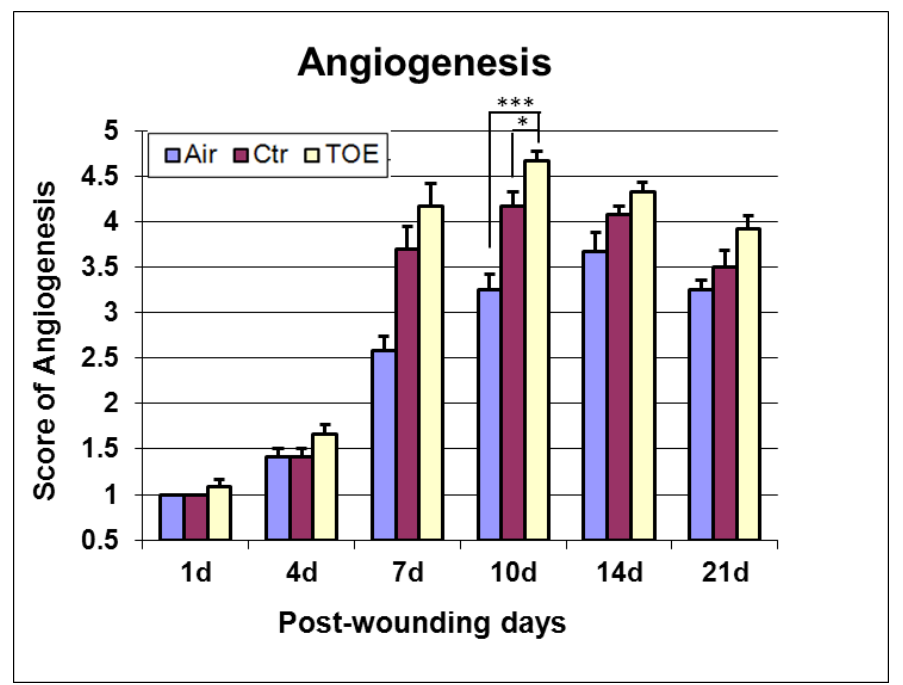

(B)

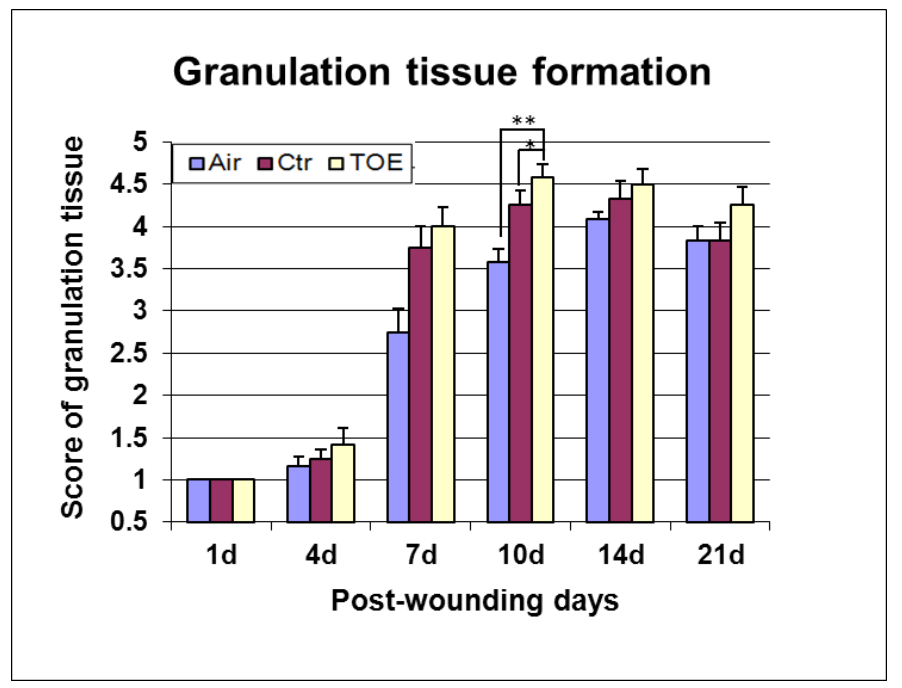




\section{Figure 2}

(2A)

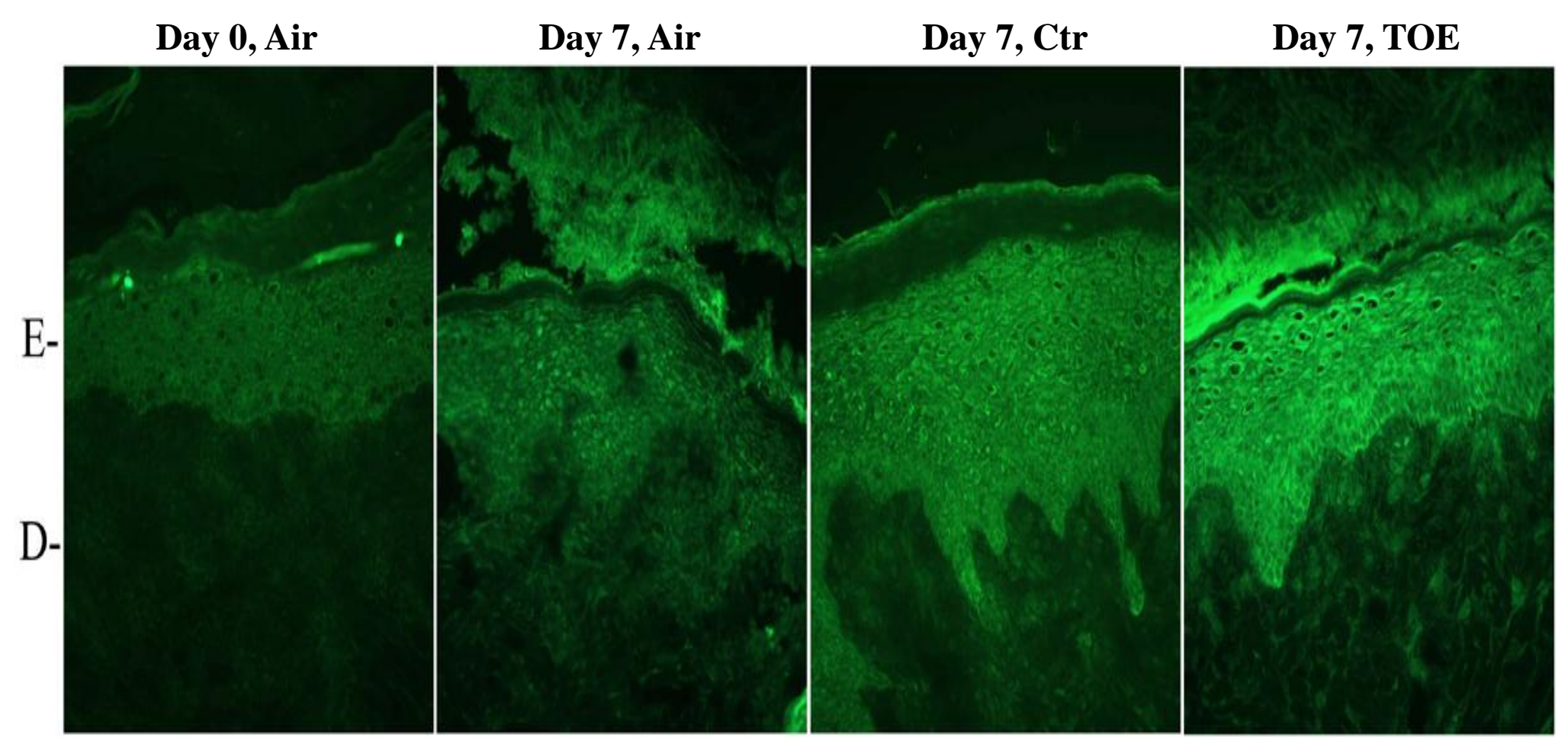


(2B)

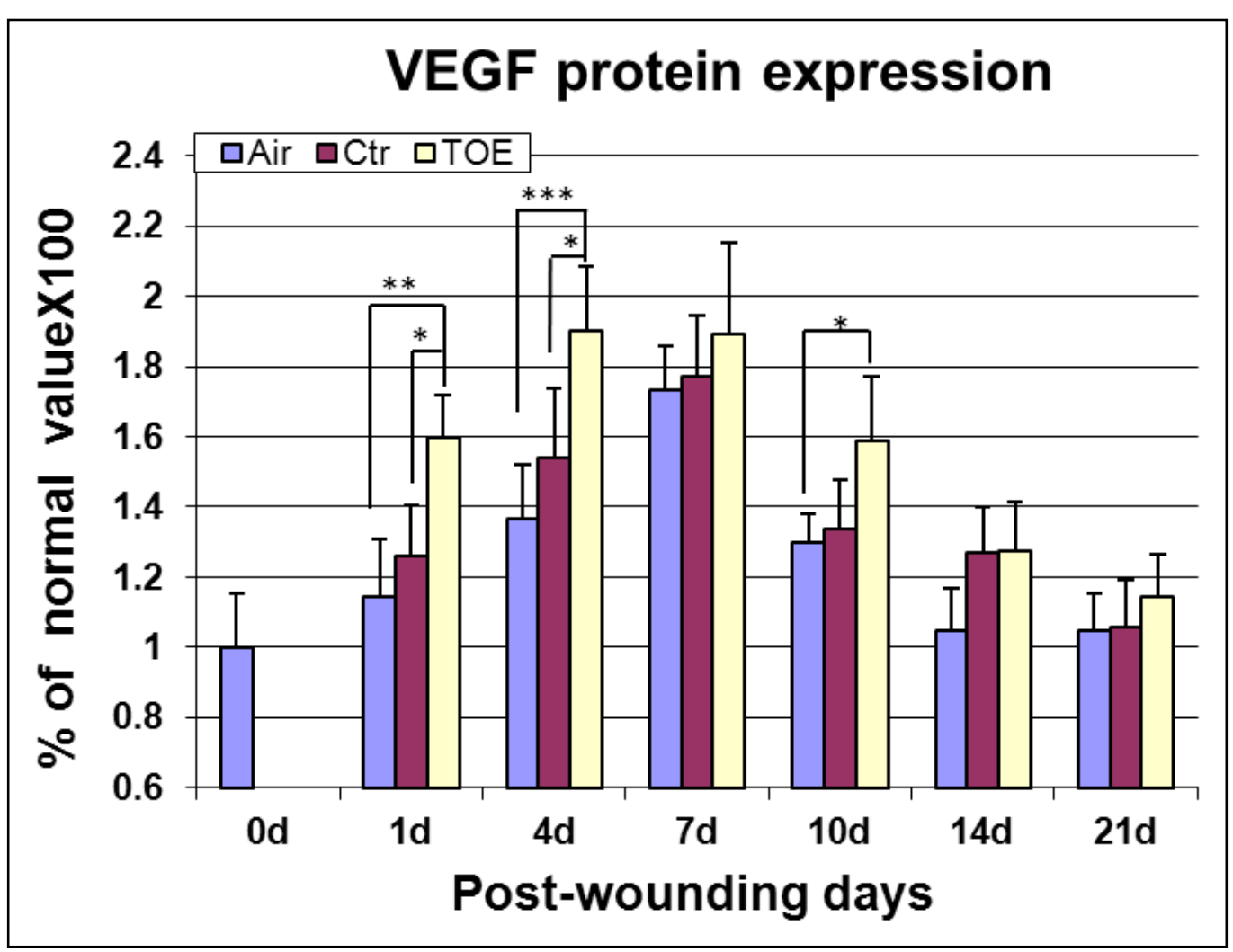


(2C)
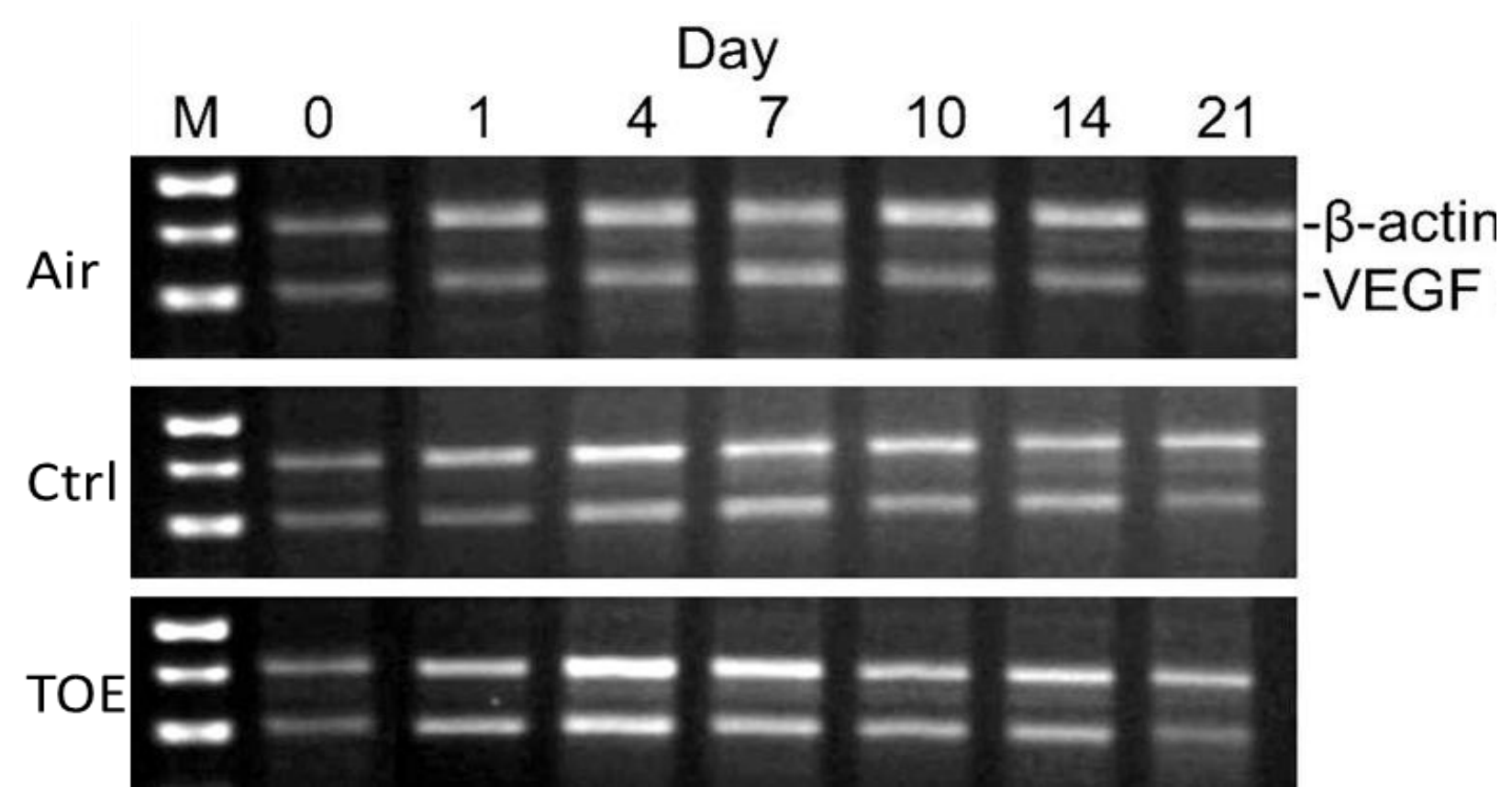
(2D)

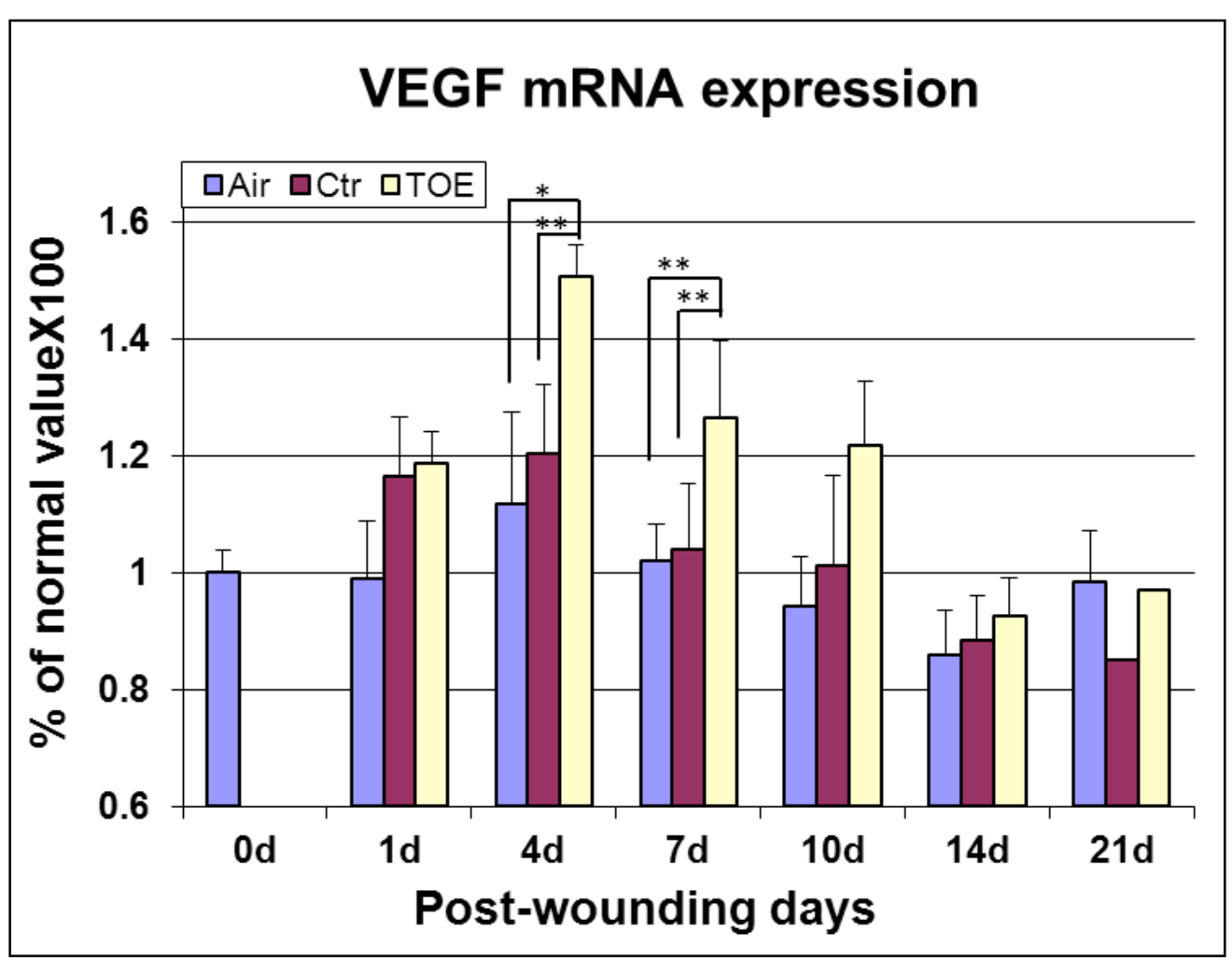


Figure 3

(3A)

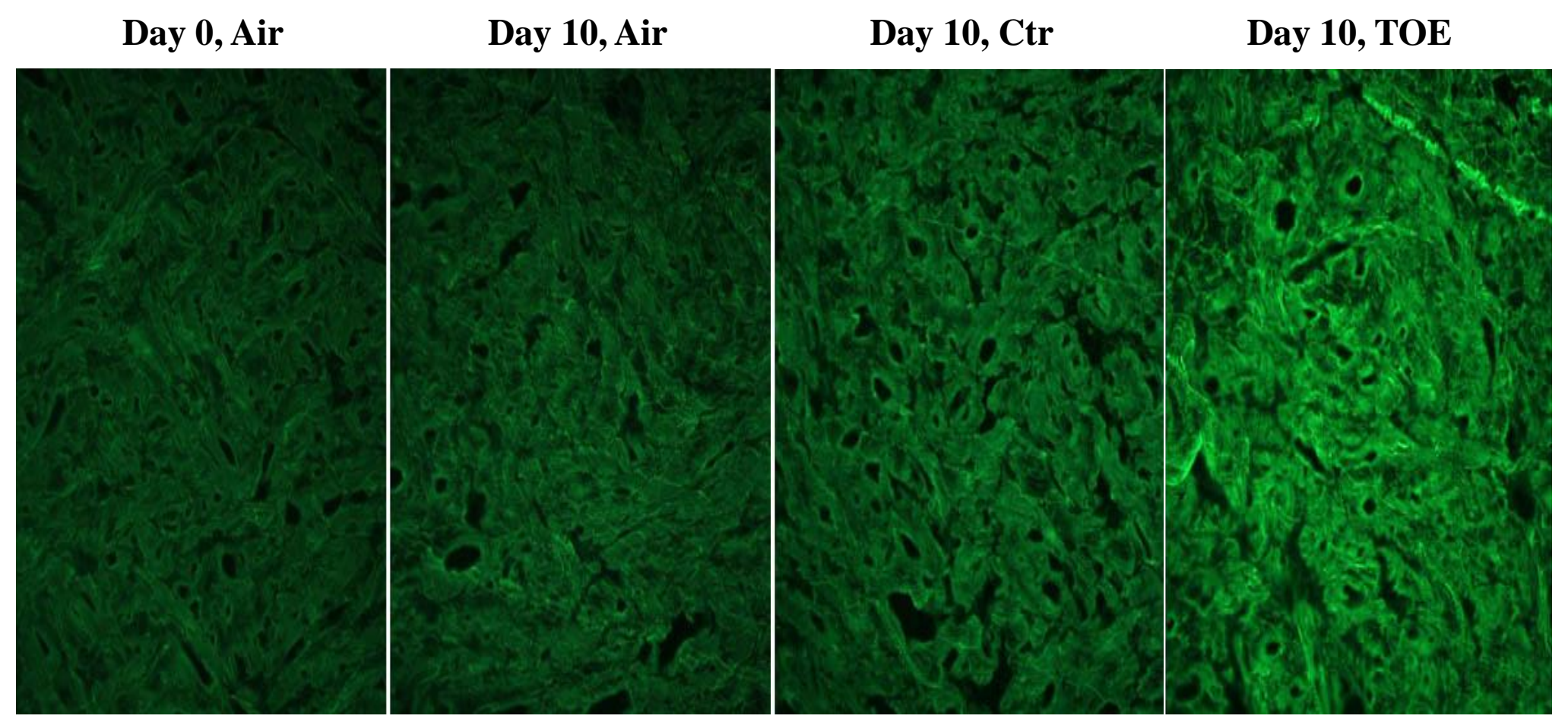


(3B)

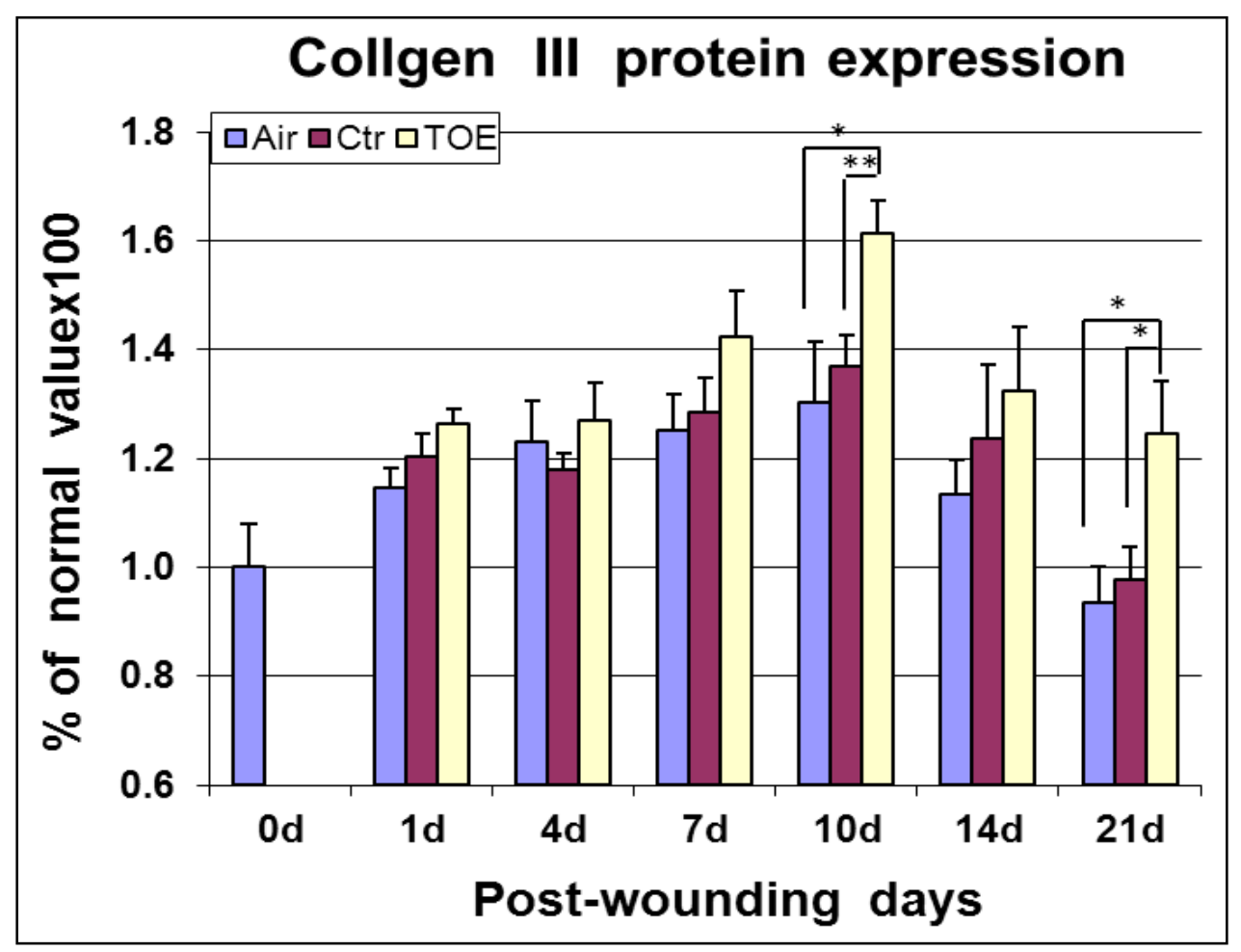


(3C)

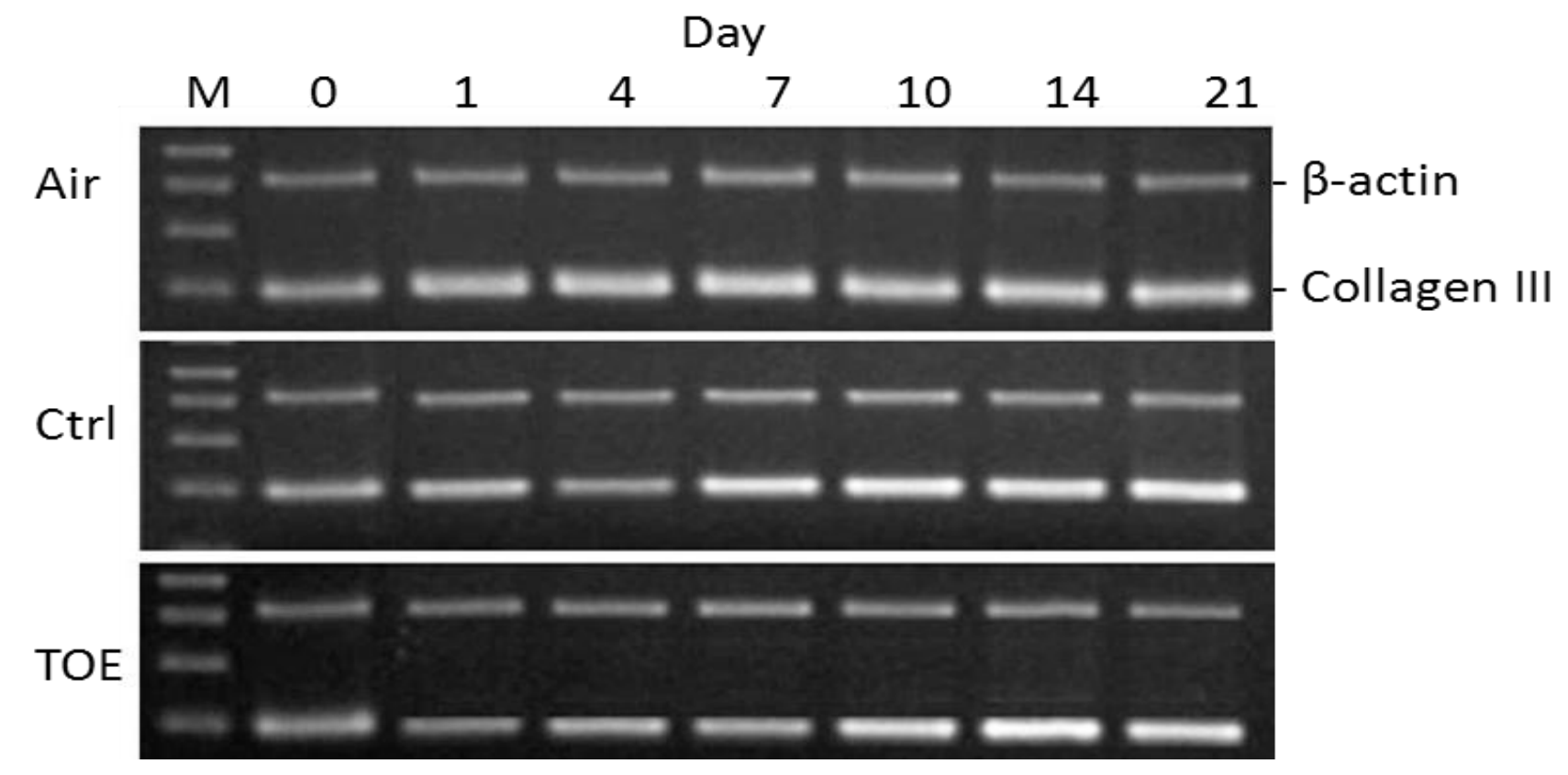


(3D)

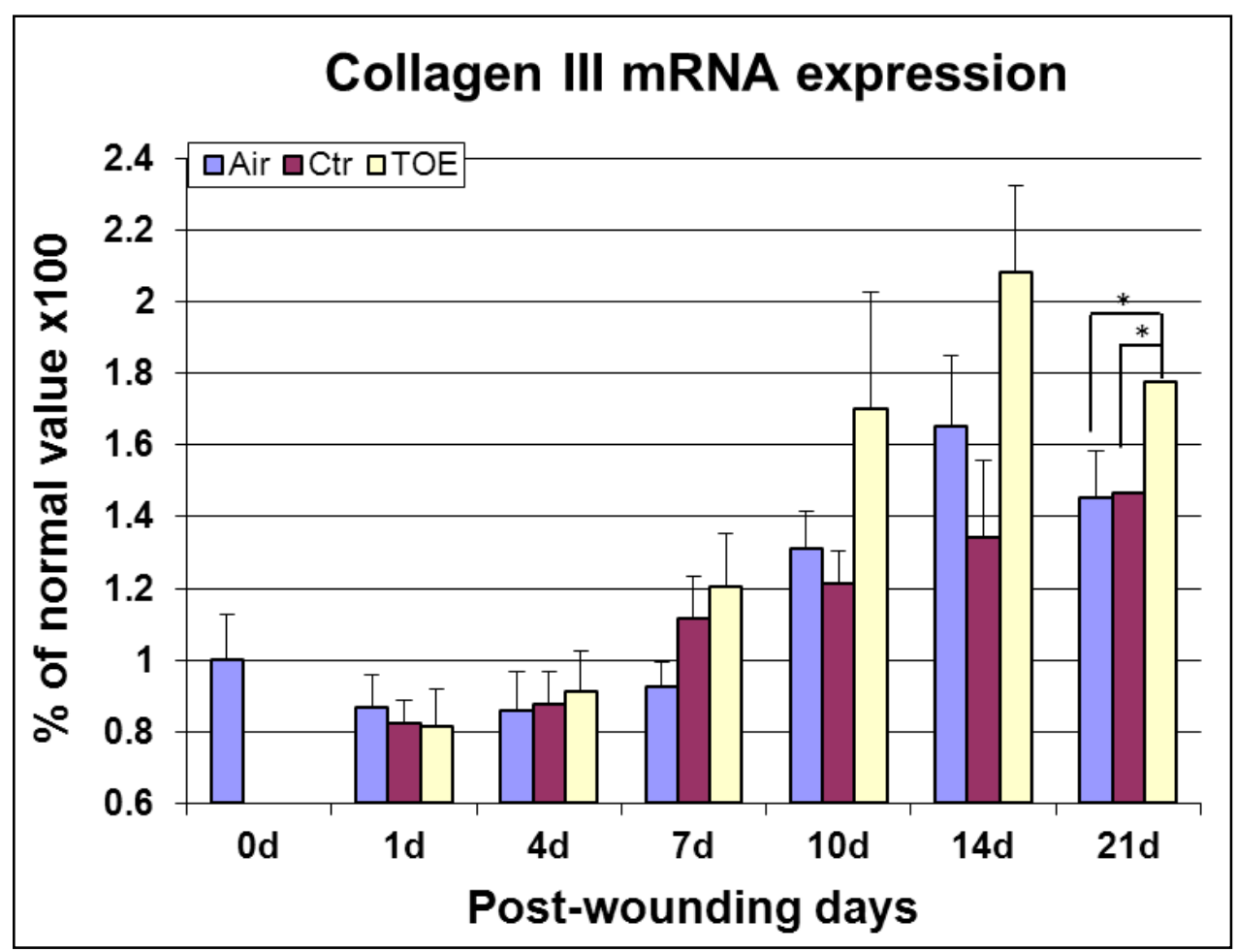


Table 1. PCR reaction primers used in the study

\begin{tabular}{|l|l|l|l|l|l|}
\hline Primers & & Sequence & BP & Cycles & Source \\
\hline $\begin{array}{l}\text { Collagen I } \\
\alpha 2\end{array}$ & Fw & GCC ACC CAG AAT GGT ACT ACT & 352 & 34 & GI: 1380559 \\
\hline & Rv & CAG GTT GCC AGT CTC TTC ATC & & & \\
\hline Collagen III & Fw & GGT GTT GGA GGT GTG GTG GTT & 207 & 34 & GI: 13805624 \\
\hline & Rv & CTC CGC TCC AGG ATG GCA GAA & & & \\
\hline VEGF & Fw & ATG CGG ATC AAA CCT CAC C & 303 & 28 & Conklin B, 2002 \\
\hline & Rv & ATC TGG TTC CCG AAA CGC TG & & & \\
\hline$\beta$-actin & Fw & GGG AGA TCG TGC GGG ACA TCA A & 410 & $34 / 28$ & GI:476331 \\
\hline & Rv & CCC GAT CCA CAC GGA GTA CTT G & & & \\
\hline
\end{tabular}

